\title{
TLR2-Dependent Reversible Oxidation of Connexin 43 at Cys260 Modifies Electrical Coupling After Experimental Myocardial Ischemia/Reperfusion
}

\author{
Florian Jürgen Raimann ${ }^{1}$ (D) $\cdot$ Stefan Dröse $^{1} \cdot$ Erik Bonke $^{1} \cdot$ Lea Schneider $^{2} \cdot$ Elisabeth Tybl $^{1} \cdot$ Ilka Wittig $^{2}$ • \\ Juliana Heidler $^{2} \cdot$ Heinrich Heide ${ }^{2,3} \cdot$ Ivana Josipovic $^{4} \cdot$ Matthias Leisegang $^{4} \cdot$ Ralf Peter Brandes $^{4} \cdot$ Jochen Roeper $^{5}$. \\ Kai Zacharowski ${ }^{1}$. Jan Mersmann ${ }^{1}$
}

Received: 27 November 2018 / Accepted: 27 March 2019/Published online: 8 April 2019

(C) Springer Science+Business Media, LLC, part of Springer Nature 2019

\begin{abstract}
We have shown previously that during myocardial ischemia/reperfusion (MI/R), toll-like receptor 2 (TLR2) signaling regulates connexin 43 (Cx43) subcellular localization and function and dampens arrhythmia formation. We aimed to identify sites capable of TLR2-dependent redox modification within Cx43. Post-ischemic TLR2 ${ }^{--}$or wild-type (WT) mouse hearts were analyzed by OxICAT. Cx43 was mutated to exclude redox modification and transfected into HL-1 cardiomyocytes (CM) that were challenged with a TLR2 agonist. We identified Cys 260 of $\mathrm{Cx} 43$ to be susceptible to reversible oxidation MI/R; TLR2 ${ }^{-/-}$leads to reduced $\mathrm{H}_{2} \mathrm{O}_{2}$ production in post-ischemic isolated mitochondria and subsequently reduced oxidation of $\mathrm{Cx} 43$ at Cys 260 . Cx 43 was dephosphorylated in WT, while phosphorylation was preserved in TLR2 ${ }^{-/}$. Mutation of $\mathrm{Cx} 43$ (C260A) and lentiviral transfection in HL-1 CM accelerated pacemaker activity and reduced activity after TLR2 ligand stimulation. We here provide evidence for TLR2-dependent reversible oxidation of $\mathrm{Cx} 43$ at Cys260, which led to decreased $\mathrm{Cx} 43$ phosphorylation and affected CM pacemaker frequency and intercellular communication.
\end{abstract}

Keywords Myocardial ischemia $\cdot$ Reperfusion $\cdot$ Toll-like receptor $2 \cdot$ Connexin $43 \cdot$ Redox regulation

$\begin{array}{ll}\text { Abbreviations } \\ \text { bsl } & \text { Baseline } \\ \text { cSNRT } & \text { Rate-corrected SNRT } \\ \text { Cx43 } & \text { Connexin } 43\end{array}$

Associate Editor Junjie Xiao oversaw the review of this article

Florian Jürgen Raimann

Florian.Raimann@kgu.de

1 Department of Anaesthesiology, Intensive Care Medicine, and Pain Therapy, University Hospital Frankfurt, Theodor-Stern-Kai 7, 60590 Frankfurt, Germany

2 Functional Proteomics, SFB 815 Core Unit, Goethe University, Frankfurt, Germany

3 Present address: Thermo Fisher Scientific GmbH, Dreieich, Germany

4 Institute for Cardiovascular Physiology, Goethe University, Frankfurt, Germany

5 Institute of Neurophysiology, Goethe University, Frankfurt, Germany

$\begin{array}{ll}\text { ecg } & \text { Electrocardiogram } \\ \text { I/R } & \text { Ischemia and reperfusion } \\ \text { MHM } & \text { Mouse heart mitochondria } \\ \text { MI/R } & \text { Myocardial ischemia/reperfusion } \\ \text { RET } & \text { Reverse electron transfer } \\ \text { ROS } & \text { Reactive oxygen species } \\ \text { SNRT } & \text { Sinus node recovery time } \\ \text { TLR2 } & \text { Toll-like receptor 2 } \\ \text { VSV } & \text { Vesicular stomatitis virus } \\ \text { WT } & \text { Wild type }\end{array}$

\section{Introduction}

Toll-like receptor 2, a receptor of the innate immune system, mediates injury in various settings of ischemia and reperfusion (I/R). Its knockout (TLR2 ${ }^{-/}$) provides protection in animal models of hepatic, renal, and myocardial ischemia/reperfusion [1-4]. We have previously shown that in myocardial ischemia/ reperfusion $(\mathrm{MI} / \mathrm{R})$ injury, this protection not only exceeds the 
mere limitation of tissue necrosis but also provides protection against lethal arrhythmias in our murine model [5].

In sterile inflammatory conditions, $\mathrm{MI} / \mathrm{R}$ endogenous ligands activate toll-like receptor 2 (TLR2) signaling. Possible ligands are biglycan, decorin, vinculin, high-mobility group box 1 , and heat shock proteins [6]. Besides the well-described pro-inflammatory signaling cascade via MyD88, TIRAP, IRAK, TRAF6, and MAP kinases leading to nuclear translocation of transcription factors AP-1 and NFKB and subsequent release of cytokines, chemokines, and adhesion molecules, the pathway via PI3K and Akt is also involved in the mediation of myocardial tissue damage [7].

We have observed previously that $\mathrm{TLR} 2^{-/-}$mice are protected from lethal arrhythmias after $30 \mathrm{~min}$ of regional myocardial ischemia and $24 \mathrm{~h}$ of reperfusion. This was associated with a better preservation of connexin $43(\mathrm{Cx} 43)$ gap junctions within the intercalated discs. Furthermore, the absence of pathological values in parameters of heart rate turbulence was detected in telemetric electrocardiogram (ecg) recordings [5]. Cx43 gap junction or hemichannel opening and subcellular translocation are regulated mainly by serine or tyrosine phosphorylation at different sites of $\mathrm{Cx} 43$. However, reversible oxidation of cysteine residues within the molecule has emerged as another mechanism by which gap junction communication can be controlled, possibly through conformational changes or modulation of kinase interaction with the protein [8].

The objective of this study was to describe the nature of arrhythmia formation in ischemic/reperfused (wild-type) WT or TLR2 ${ }^{-/-}$mouse hearts via intracardiac ecg recordings and pacing protocols. Furthermore, we aimed to identify the generator of reactive oxygen species (ROS) along with the pattern of reversible oxidations in a redox proteomic approach in murine myocardium. Therefore, we screened especially for redox targets within the $\mathrm{Cx} 43$ molecule. Among the redox targets identified, we here identified one particular cysteine residue (Cys260) of Cx43 to investigate whether this differential oxidation is critical for action potential generation and propagation between cardiomyocytes.

\section{Methods}

\section{Animals}

All experiments were performed in accordance with the regional animal welfare committee (Regierungspräsidium Darmstadt) and in accordance with the guidelines from Directive 2010/63/EU of the European Parliament on the protection of animals used for scientific purposes. Ten to 14week-old C57BL/6JRj (Janvier Labs, Le Genest-Saint-Isle, France) WT and TLR2 ${ }^{--}$(B6.129-TLR2tm $1 \mathrm{kir} / \mathrm{J}$, The Jackson Laboratories, Bar Harbor, ME, USA) mice were used.
Animals were sacrificed at the end of the experiment under deep sedation with pentobarbital $(80 \mathrm{mg} / \mathrm{kg}$, i.p. $)$ and buprenorphine $(0.1 \mathrm{mg} / \mathrm{kg}$, s.c.) by excision of the heart and terminal bleeding.

$n=6 /$ group animals were used for electrophysiology/ pacemaker recordings, $n=6$ /group animals were used for mitochondria isolation, and $n=3$ /group animals were used for OxICAT.

\section{Myocardial Ischemia/Reperfusion}

$\mathrm{MI} / \mathrm{R}$ was performed as previously described [2]. After anesthesia induction with pentobarbital $(80 \mathrm{mg} / \mathrm{kg}$, i.p.) and buprenorphine $(0.1 \mathrm{mg} / \mathrm{kg}$, s.c. $)$, a tracheotomy was performed and animals were connected to a ventilator $\left(\mathrm{V}_{\mathrm{T}}\right.$ $9 \mathrm{~mL} / \mathrm{kg}, 110$ strokes $/ \mathrm{min}, \mathrm{F}_{\mathrm{i}} \mathrm{O}_{2}$ 0.5). After left thoracotomy the left anterior descending coronary artery was ligated for $30 \mathrm{~min}$, followed by reperfusion. Tissue was harvested at the end of ischemia without reperfusion (OxICAT), after $2 \mathrm{~min}$ of reperfusion (mitochondria isolation, OxICAT), or $40 \mathrm{~min}$ of reperfusion (electrophysiology).

\section{Electrophysiology}

A 1.1F octopolar catheter (Transonic Scisense, London, Ontario, Canada), which was connected to an Octal Bio Amp and a PowerLab (ADInstruments, Oxford, UK), was inserted via the right jugular vein. Its correct position was verified by identification of typical intracardiac ecg traces. After determination of atrial and ventricular pacing threshold, a preset pacing protocol lasting $7 \mathrm{~min}$ was started at each time point using twice the threshold energy. Analysis was performed at baseline (bsl), after $15 \mathrm{~min}$ of ischemia, $10 \mathrm{~min}$ reperfusion, and $30 \mathrm{~min}$ reperfusion. Intracardiac and threelead surface ecg traces were recorded to determine heart rate (beats/min), cycle length (RR interval in $\mathrm{ms}$ ), PR (ms), QRS (ms), QT, and rate-corrected QT (modified Bazett formula, $\mathrm{ms}$ ), as previously described [9]. Twenty beats per time point were averaged and examined by two independent investigators. After recording of spontaneous ecg activity, programmed stimulation was performed using a stimulus generator (STG2000, Multichannel systems, Reutlingen, Germany) via the two most proximal electrodes situated in the atrium and afterwards via the two most distal electrodes situated in the ventricle. Sinus node recovery time (SNRT) was measured in triplicate after 30 atrial stimuli at 120-, 100-, and 80-ms cycle length. Rate-corrected SNRT (cSNRT) was retrieved by subtracting spontaneous cycle length from SNRT. Wenckebach periodicity was determined by eight atrial stimuli starting at $120-\mathrm{ms}$ cycle length and further shortening by $2 \mathrm{~ms}$. Ventricular vulnerability was tested by eight stimuli at $120-\mathrm{ms}$ cycle length followed by three extra stimuli with decreasing cycle length between 60 and $20 \mathrm{~ms}$. Afterwards, ventricular 
burst stimulation was performed with cycle lengths of 50, 40, 30, 20, and $10 \mathrm{~ms}$ lasting $5 \mathrm{~s}$. Energy applied was twice the pacing threshold. Ventricular tachycardia was defined as $\geq 4$ ventricular ectopic beats. ECG traces were analyzed with LabCHART software (ADInstruments, Oxford, UK).

\section{Cx43 Western Blot}

Murine heart tissue was lysed in ice-cold lysis buffer $(150 \mathrm{mM}$ $\mathrm{NaCl} ; 50$ mM Tris-HCl, pH 7.4; 1 mM EDTA; 1 mM PMSF; $1 \%$ Triton X-100; $1 \%$ sodium deoxycholate; $0.1 \%$ SDS; $5 \mu \mathrm{g} /$ $\mathrm{mL}$ aprotinin; $5 \mu \mathrm{g} / \mathrm{mL}$ leupeptin). After centrifugation (20 $\mathrm{min} ; 10,000 \times g$ ), the supernatants were removed and total protein concentration was determined (bicinchoninic acid method). The appropriate amount of loading buffer $(6 \mathrm{~mL}$ deionized $\mathrm{H}_{2} \mathrm{O} ; 1 \mathrm{~mL} 500 \mathrm{mM}$ Tris- $\mathrm{HCl}, \mathrm{pH} 6.8 ; 2 \mathrm{~mL}$ glycerol; $0.1 \mathrm{~g}$ SDS; $5 \mathrm{mg}$ bromophenol blue; $1 \mathrm{~mL} \beta$ mercaptoethanol) was added. Samples were loaded, separated by SDS-PAGE and transferred onto PVDF membranes. The blots were incubated with the appropriate primary antibody, Connexin 43/Gjal (abcam, ab79010), diluted 1:6000 in 5\% BSA in TBS-T, and secondary antibody (R\&D Systems; HAF007; 1:1000). Signals were detected by enhanced chemiluminescence (Santa Cruz, sc-2048) and normalized to $\beta$ actin (Santa Cruz, sc-47778).

\section{Isolation of Mouse Heart Mitochondria}

Mouse heart mitochondria (MHM) were isolated following the protocol developed for rat heart mitochondria [10] with the modification that all buffer volumes and trypsin and trypsin inhibitor quantities were halved. Diced ventricular tissue was minced and washed with a solution containing $300 \mathrm{mM}$ sucrose, $10 \mathrm{mM} \mathrm{Na}^{+} /$HEPES (pH 7.2), and $0.2 \mathrm{mM}$ EDTA. The tissue was treated with trypsin $(\sim 0.1 \mathrm{mg} / \mathrm{mL})$ for $15 \mathrm{~min}$ and homogenized twice before adding soybean trypsin inhibitor $(\sim 0.3 \mathrm{mg} / \mathrm{mL})$. The heart mitochondria were subsequently washed, centrifuged, and suspended in a solution containing $300 \mathrm{mM}$ sucrose, $10 \mathrm{mM} \mathrm{Na}^{+} /$HEPES (pH 7.4), $0.2 \mathrm{mM}$ EDTA, and $1 \mathrm{mg} / \mathrm{mL}$ fatty acid free bovine serum albumin.

\section{Detection of Reactive Oxygen Species}

ROS were detected by the Amplex Red/HRP assay in fluorescence mode as described by Bleier et al. [11]. The assay was calibrated with known $\mathrm{H}_{2} \mathrm{O}_{2}$ concentrations $(0-5 \mu \mathrm{M})$. Resorufin formation was measured at $30^{\circ} \mathrm{C}$ in a SpectraMax $\mathrm{M} 2{ }^{\mathrm{e}}$ multimode reader (Molecular Devices, Germany) at the following settings: sensitivity low; top read; excitation $540 \mathrm{~nm}$, emission $600 \mathrm{~nm}$ (fixed bandwidth $9 \mathrm{~nm}$ for excitation/emission); cutoff filter $590 \mathrm{~nm}$. MHM were diluted in a reaction mixture containing $50 \mu \mathrm{M}$ Amplex Red, $0.1 \mathrm{U} / \mathrm{mL}$ HRP, $200 \mathrm{mM}$ sucrose, $10 \mathrm{mM}$ Tris/HCl, $10 \mathrm{mM}$ potassium phosphate (pH 7.0), $10 \mathrm{mM} \mathrm{MgSO}_{4}, 100 \mu \mathrm{M}$ ATP, and $2 \mathrm{mM}$ EDTA. MHM were fueled by $5 \mathrm{mM}$ succinate. Inhibitors/ effectors were added in the following final concentrations (if not indicated otherwise): $1 \mu \mathrm{M}$ antimycin $\mathrm{A}, 1 \mu \mathrm{M}$ stigmatellin, $50 \mathrm{nM}$ FCCP, $250 \mathrm{nM}$ atpenin A5, and $2 \mu \mathrm{g} /$ $\mathrm{mL}$ oligomycin.

\section{Redox Proteomics}

Sample Preparation To quantitatively identify redox-modified cysteines in the myocardium, the OxICAT protocol by Leichert et al. [12] was used with some adaption to mouse heart tissue. A cleavable ICAT method development kit and cleavable ICAT reagent kit were obtained from AB SCIEX (Darmstadt, Germany). Tissue was excised; the ischemic region was quickly separated from the non-ischemic region of the left ventricle and was snap-frozen in liquid nitrogen. Samples were weighed and homogenized in ice-cold $10 \%$ trichloroacetic acid (TCA), and a volume corresponding to $5 \mathrm{mg}$ total tissue was used for further analysis. Samples were centrifuged for $30 \mathrm{~min}\left(13,000 \times \mathrm{g}, 4{ }^{\circ} \mathrm{C}\right)$, and TCA precipitates were consecutively washed in $10 \%$ and $5 \%$ ice-cold TCA. Resulting pellets were dissolved in denaturing alkylation buffer (DAB; $8 \mathrm{M}$ urea, $10 \mathrm{mM}$ EDTA, $200 \mathrm{mM}$ Tris$\mathrm{HCl}, \mathrm{pH} 8.5$ ) containing $0.5 \%$ SDS. Light ICAT reagent vials were dissolved in $10 \mu \mathrm{L}$ acetonitrile and $5 \mu \mathrm{L}$ was transferred to each sample. Samples were incubated for $75 \mathrm{~min}$ at $37{ }^{\circ} \mathrm{C}$ and $1300 \mathrm{rpm}$ in the dark. The light ICAT reagent was removed by precipitation of proteins with ice-cold acetone overnight at $-20^{\circ} \mathrm{C}$. The samples were then washed twice with ice-cold acetone, dissolved in $50 \mu \mathrm{L}$ DAB buffer and reduced with $1 \mu \mathrm{L}$ of reducing reagent $(50 \mathrm{mM}$ tris(2carboxyethyl)phosphine (TCEP)) provided in the kit for $5 \mathrm{~min}$ at $37^{\circ} \mathrm{C}$ and $1300 \mathrm{rpm}$. Then, the contents of 0.5 vials of the heavy ICAT reagent dissolved in acetonitrile were added, and samples were incubated for $2 \mathrm{~h}$ at $37^{\circ} \mathrm{C}$ and $1300 \mathrm{rpm}$ in the dark. Samples were digested with trypsin for $16 \mathrm{~h}$ at $37^{\circ} \mathrm{C}$. Peptides were acidified by adding $20 \mu \mathrm{L}$ pure formic acid and purified by solid phase extraction (SPE) cartridges (Empore ${ }^{\mathrm{TM}} 4115$ (SD), St. Paul, USA). Enrichment of ICAT-labeled peptides on avidin columns and cleavage of biotin moiety from the isotope-coded tag were done as described in the manufacturers' manual.

LC-MS/MS Analysis LC/MS was performed on a Thermo Scientific ${ }^{\mathrm{TM}}$ Orbitrap XL mass spectrometer with an Agilent 1200 nano-HPLC (high-performance liquid chromatography) at the front end. Peptides were resolved in $4 \%$ acetonitrile and $0.5 \%$ formic acid and loaded on a C18 reversed-phase precolumn (Zorbax 300SB-C18, Agilent Technologies, Palo Alto, USA) followed by separation on in-house-packed 3- $\mu \mathrm{m}$ Reprosil C18 resin (Dr. Maisch $\mathrm{GmbH}$ ) picotip emitter tip (diameter $75 \mu \mathrm{m}$, 
length $10 \mathrm{~cm}$, New Objectives) using a gradient from 5\% acetonitrile and $0.1 \%$ formic acid to $50 \%$ acetonitrile and $0.1 \%$ formic acid for $90 \mathrm{~min}$ with a flow rate of $200 \mathrm{~nL} /$ min. MS data were recorded by data-dependent top 10 acquisition selecting the 10 most abundant precursor ions in positive mode for fragmentation using dynamic exclusion of $3 \mathrm{~min}$. Only higher charged ions (2+) were selected for MS/MS scans in the linear ion trap by CID at $35 \%$ collision energy. Lock mass option for $\mathrm{m} / \mathrm{z} 445.120025$ was enabled to ensure high mass accuracy during many following runs.

Data Analysis MS Data were searched using Mascot Server (version 2.2) against a database containing speciesspecific (Mus musculus) protein sequences downloaded from www.uniprot.org. The Mascot search settings were as follows: maximum missed cleavages 1 , precursor mass tolerance $10 \mathrm{ppm}$, fragment ion tolerance $0.8 \mathrm{Da}$, and optional modifications allowed on methionine (oxidation) and cysteine. Only peptides with individual ion scores indicating identity $(P<0.05)$ were considered significant. Quantification of heavy/light ICAT ratios of peptides was performed by Proteome Discoverer 1.2. Peptides containing cysteines were selected and oxidation status (\%) was calculated and further statistically analyzed.

\section{Cloning of pHAGE-CMV-mmGja1 and pHAGE-CMV-mmGja1C260A Lentiviral Constructs}

Full-length protein-coding regions of $\mathrm{mmGjal}$ and mmGja1-C260A were PCR amplified with Phusion High-Fidelity DNA Polymerase (F530L, Thermo Fisher) from pCMV6-Kan/Neo-Gja1ORF (MC205621, Origene) with the primers $5^{\prime}$-ATTTGCGGCCGCACCATGGG TGACTGGAGCGCCTTGGGG-3' (forward) and 5'GGGTATCGATTTAAATCTCCAGGTCATCAGGC CGAGGTC-3' (reverse). Afterwards, the PCR products were digested with ClaI/NotI and ligated into pHAGECMV-dsRed-UBC-GFP-W, which was a gift from Darrell Kotton (Addgene plasmid \#24526) [13].

\section{Lentiviral Transfection of HL-1 Cardiac Muscle Cells}

Vesicular stomatitis virus (VSV)-pseudotyped lentivirus was produced by transfection of human embryonic kidney 293T cells with the lentiviral constructs in combination with HGPM2, Tat1B, Rev1B, and VSV-G, which were a gift from Bianling Liu. Viral supernatants were collected, concentrated, and snap-frozen after 4 days. Transfection of $70 \%$ subconfluent HL-1 cardiac muscle cells with mmGjal wildtype and mmGja1-C260A viral particles was performed for 10 days.
Multielectrode Array Recordings of HL-1 Cells

HL-1 cells were cultured in Claycomb medium as previously described [14] on multielectrode arrays until confluency was reached. Detection of spontaneous action potential generation was started $10 \mathrm{~min}$ after transfer of the multielectrode array (MEA) from the incubator to the MEA-Headstage and was continuously recorded during exposure to $\mathrm{H}_{2} \mathrm{O}_{2}(0.1 \mu \mathrm{M})$ or TLR2 ligand $\mathrm{Pam}_{3} \mathrm{CSK}_{4}(10 \mu \mathrm{g} / \mathrm{mL})$.

\section{Patch-Clamp Recordings of Transfected HL-1 Atrial Cells}

Whole-cell patch-clamp recordings of individual HL-1 atrial cells in cultures transfected with either wild-type or mutant Cx43 were carried out in a current clamp under visual control (Zeiss Axioskop FS) using an HEKA EPC-10 patch-clamp amplifier (HEKA, Lambrecht/Pfalz, Germany). HL-1 cultures on coverslips were transferred to a recording chamber and continuously perfused $(2 \mathrm{~mL} / \mathrm{min})$ with an external Tyrode's solution containing (in $\mathrm{mM}$ ) the following: $5.4 \mathrm{KCl}, 137$ $\mathrm{NaCl}, 1.8 \mathrm{CaCl}_{2}, 0.5 \mathrm{MgCl}_{2}, 10$ HEPES (adjusted to $\mathrm{pH}=$ 7.4 with $\mathrm{NaOH}$ ), and 11 glucose at $36{ }^{\circ} \mathrm{C}$. Coverslips were superfused for $>10 \mathrm{~min}$ before patching and recorded for $>$ 5 min before bath-perfused with Tyrode's solution containing $10 \mu \mathrm{g} / \mathrm{mL}$ PAM-cys to activate TLRs. A patch pipette (borosilicate glass, tip resistance 4-5 MQ) contained (in $\mathrm{mM}$ ) the following: $135 \mathrm{~K}$-gluconate, $5 \mathrm{KCl}, 10 \mathrm{HEPES}, 0.1 \mathrm{EGTA}, 2$ $\mathrm{MgCl}_{2}, 0.2 \mathrm{Li}_{2} \mathrm{GTP}, 2 \mathrm{Na}_{2} \mathrm{ATP}, \mathrm{pH}=7.35$ with $\mathrm{KOH}(270$ $300 \mathrm{mOsm}$ ), and $1 \mathrm{mg} / \mathrm{mL}$ neurobiotin for post hoc visualization. Signals were digitized at $20 \mathrm{kHz}$ and logged onto a personal computer with the Patchmaster (HEKA) software. Data were analyzed using Fitmaster (HEKA) and IGOR (WaveMetrics) software. After recording, HL-1 atrial cultures were fixed for $2 \mathrm{~h}$ in $4 \%$ PFA and $0.2 \mathrm{M}$ PBS, washed $4 \times$ for $10 \mathrm{~min}$ in PBS, incubated for $1 \mathrm{~h}$ in blocking solution $(0.2 \mathrm{M}$ PBS with $10 \%$ horse serum, $0.5 \%$ Triton X-100, and $0.2 \%$ BSA), and incubated overnight in carrier solution containing Streptavidin Alexa Fluor 488 (1:1000; Molecular Probes). Sections were rinsed in PBS and mounted on slides (Vectashield, Vector Laboratories). Fluorescent stainings of filled cells were analyzed using a laser scanning microscope (Nikon Eclipse 90i, Nikon GmbH).

\section{Statistics}

Results are presented as mean \pm SEM of $n$ observations. Comparisons were made using Prism 5.0 (GraphPad Software Inc., La Jolla, CA). After normality testing, data were analyzed using Student's $t$ test or ANOVA/Bonferroni for multiple comparisons. $P$ values $<0.05$ were considered significant. 


\section{Results}

\section{Electrophysiology}

No significant differences in basal cardiac electrophysiology were detected between WT and TLR2 ${ }^{-1}$ at baseline (Table 1 , Fig. 1). Ventricular tachycardia (VT) was not inducible at baseline or during ischemia or reperfusion. Neither sinus cycle length (SCL), QTc, SNRT, nor Wenckebach periodicity showed significant differences between time points or study groups. PR and QRS intervals, however, were significantly shorter in TLR2 ${ }^{-/}$animals at late reperfusion (30 min) compared with WT (Table 1, Fig. 1b,c).

\section{Action Potential Propagation in HL-1 Cells Is Accelerated by TLR2 or ROS Stimulation}

Spontaneously beating HL-1 cardiomyocytes grown in monolayers on multielectrode arrays were stimulated with TLR2 agonist $\mathrm{Pam}_{3} \mathrm{CSK}_{4}(10 \mu \mathrm{g} / \mathrm{mL})$ which induced acceleration of AP propagation within $30 \mathrm{~min}$ (Fig. 1e). Likewise, administration of $\mathrm{H}_{2} \mathrm{O}_{2}$ to the culture medium (end concentration $0.1 \mu \mathrm{M}$ ) induced a transient acceleration of propagation velocity within the first $10 \mathrm{~min}$, which returned to baseline after 20 min (Fig. 1f). Figure 1g shows an example of a spreading action potential over the HL-1 cardiomyocyte monolayer.

\section{Cysteine Residue Cys260 of Cx43 Is Less Frequently Oxidized in Post-Ischemic TLR2 ${ }^{-1-}$ Hearts}

OxICAT results of murine cardiac samples subjected to MI/R were screened for $\mathrm{Cx} 43$ peptides. This approach retrieved a peptide containing Cys260, which was subject to redox regulation. Reversible oxidation was significantly more frequent in hearts of WT compared with TLR2 ${ }^{-/-}$mice after $\mathrm{MI} / \mathrm{R}$ in both the post-ischemic and the remote region (Fig. 2a).

\section{$\mathrm{H}_{2} \mathrm{O}_{2}$ Production by Reverse Electron Transfer at Complex I Is Reduced in Mitochondria Isolated from $\mathrm{TLR}^{-1-}$ Hearts}

Mitochondria were isolated from hearts that underwent $30 \mathrm{~min}$ ischemia followed by $2 \mathrm{~min}$ reperfusion in vivo. Mitochondria were separately isolated from remote and postischemic myocardium. It is well known that succinate accumulates in heart mitochondria during ischemia [15] and that this drives a massive oxidative burst at complex I by reverse electron transfer (RET) during reperfusion [15-17]. We detected a significant $\mathrm{H}_{2} \mathrm{O}_{2}$ emission in presence of $5 \mathrm{mM}$ succinate and $100 \mu \mathrm{M}$ ATP/ADP in WT mitochondria (Fig. 2b) that was further increased by the addition of the complex $\mathrm{V}$ inhibitor oligomycin. Inhibition of complex $\mathrm{V}$ increases the membrane potential and hence the driving force for RET [18]. This $\mathrm{H}_{2} \mathrm{O}_{2}$ production/emission was completely suppressed by the electrogenic protonophore FCCP that uncouples the membrane potential and by the $\mathrm{II}_{\mathrm{Q}}$ site (ubiquinone binding site of complex II) inhibitor atpenin A5, indicating that no other generator than complex I was involved under these conditions. In presence of atpenin $\mathrm{A} 5$, site $\mathrm{II}_{\mathrm{F}}$ (flavin site of complex II) can produce ROS, but this is suppressed by high succinate concentrations $[19,20]$. Both the "basal" and the oligomycin-induced $\mathrm{H}_{2} \mathrm{O}_{2}$ emission were reduced to $\sim 50 \%$ in mitochondria from $\mathrm{TLR}^{-/-}$mice, indicating that they were emitting/producing less of the damaging ROS during conditions that mimic ischemia/reperfusion. It needs to be

Table 1 Electrophysiology parameters during the course of MI/R

\begin{tabular}{|c|c|c|c|c|c|c|c|c|}
\hline \multirow[b]{2}{*}{ ECG parameters } & \multicolumn{4}{|l|}{ WT } & \multicolumn{4}{|c|}{$\mathrm{TLR}^{-/-}$} \\
\hline & Bsl & $15 \mathrm{~min}$ ischemia & 10 min reperfusion & 30 min reperfusion & Bsl & $15 \mathrm{~min}$ ischemia & $10 \mathrm{~min}$ reperfusion & 30 min reperfusion \\
\hline $\mathrm{SCL}(\mathrm{ms})$ & 185 & 186 & 192 & 187 & 180 & 193 & 201 & 193 \\
\hline PR (ms) & 40 & 39 & 39 & 43 & 39 & 42 & 39 & $27^{*}$ \\
\hline QRS (ms) & 13 & 12 & 12 & 13 & 13 & 12 & 11 & $11^{*}$ \\
\hline QTc (ms) & 23 & 28 & 22 & 25 & 30 & 21 & 20 & 23 \\
\hline SNRT (120 ms) & 214 & 219 & 220 & 242 & 213 & 233 & 232 & 229 \\
\hline SNRT (100 ms) & 217 & 208 & 217 & 227 & 203 & 207 & 223 & 217 \\
\hline SNRT (80 ms) & 213 & 196 & 229 & 211 & 208 & 210 & 227 & 194 \\
\hline cSNRT (100 ms) & 33 & 22 & 24 & 40 & 23 & 14 & 22 & 25 \\
\hline $\mathrm{SNRT} / \mathrm{SCL} \times 100$ & 118 & 112 & 112 & 123 & 113 & 106 & 111 & 126 \\
\hline Wenckebach & 114 & 116 & 115 & 117 & 109 & 108 & 114 & 114 \\
\hline $\mathrm{VT}(n)$ & 0 & 0 & 0 & 0 & 0 & 0 & 0 & 0 \\
\hline
\end{tabular}

$S C L$ sinus cycle length, $S N R T$ sinus node recovery time, $c S N R T$ rate-corrected SNRT, $V T$ ventricular tachycardia

$n=6 /$ group, repeated measures ANOVA/Bonferroni, $* P<0.05 \mathrm{WT}$ vs. TLR2 ${ }^{-/-}$ 

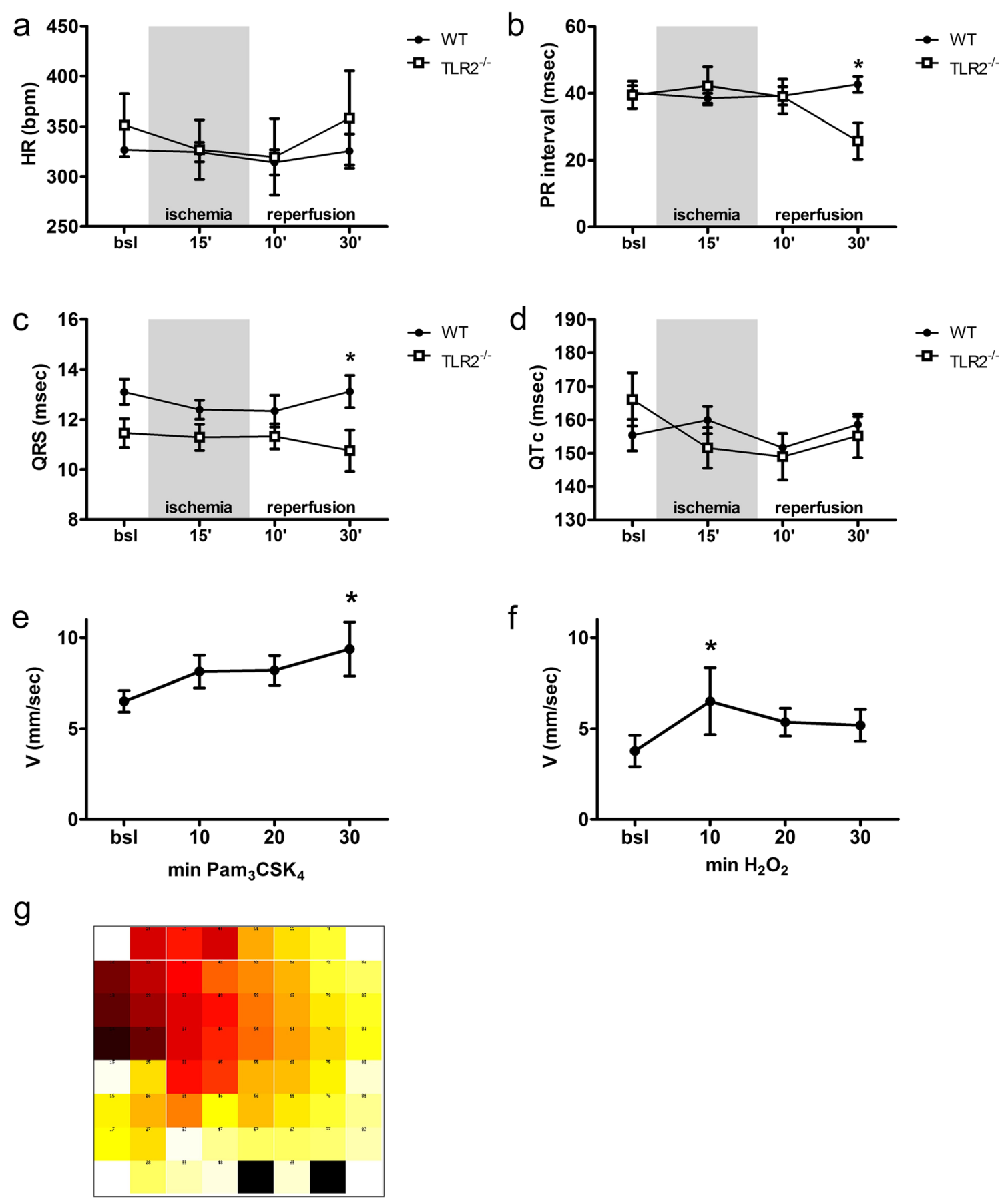

Fig. 1 In vivo/in vitro electrophysiology. A standard pacing protocol was applied after preparation, prior to LAD ligation (bsl), after $15 \mathrm{~min}$ of ischemia, and after 10 and $30 \mathrm{~min}$ of reperfusion. No significant differences at bsl were detected (a-d). PR (b) and QRS (c) were significantly shorter in TLR2 ${ }^{-/-}$compared with WT at late reperfusion ( $n=6$ /group,

emphasized that the Amplex Red/HRP assay is only detecting $\mathrm{H}_{2} \mathrm{O}_{2}$ that escapes the mitochondrial antioxidant systems, and a reduced emission can mean either a reduced production or an increased scavenging by the antioxidant systems or a combination of both. Nevertheless, the general differences between WT and TLR2 ${ }^{-/}$were still obvious. In presence of
$* P<0.05)$. Multielectrode arrays were performed in spontaneously beating HL-1 cardiomyocytes. Conduction velocity increases after stimulation with TLR2 agonist $\mathrm{Pam}_{3} \mathrm{CSK}_{4}(n=5, \mathbf{e})$ or $\mathrm{H}_{2} \mathrm{O}_{2}(n=5, \mathbf{f})$, $* P<0.05$. Example showing spread of the action potential over the HL1 cardiomyocyte monolayer $(\mathbf{g})$

the Qi site inhibitor antimycin A, the membrane potential is largely distorted, the RET does not occur, and the ROS production can be largely attributed to the Qo site of complex III (site $\mathrm{III}_{\mathrm{Qo}}$ ) $[16,21]$. However, there was no difference in the ability to produce superoxide at site $\mathrm{III}_{\mathrm{Qo}}$ between mitochondria from WT and TLR2 ${ }^{-/}$mice. Administration of the site 
a

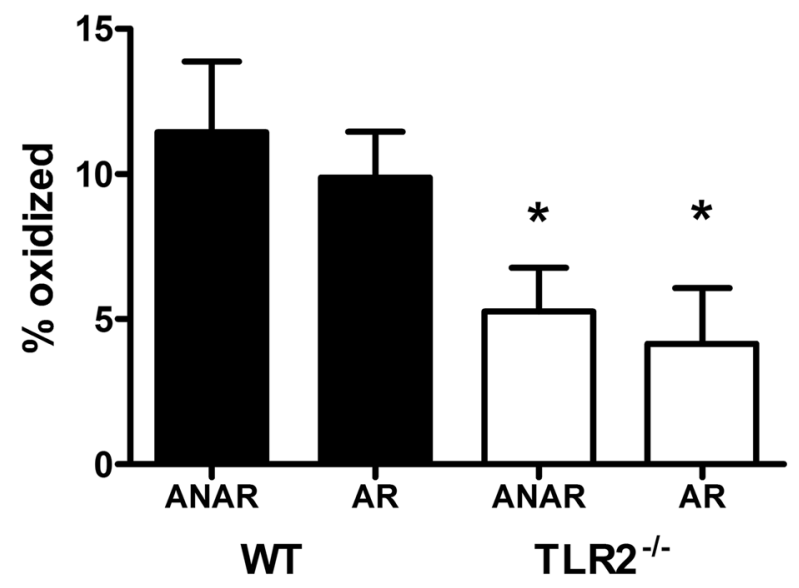

b

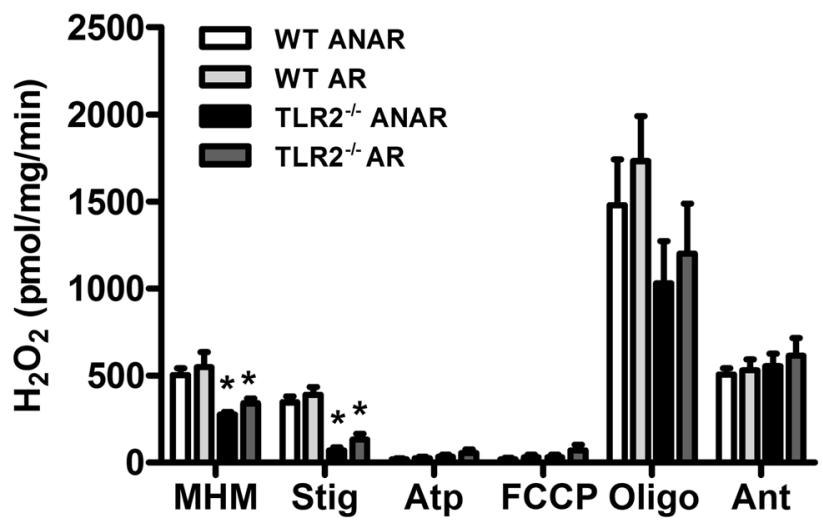

Fig. 2 OxICAT identifies significantly reduced reversible oxidation of Cx43 Cys260 in $\mathrm{TLR}^{-/-}$post-ischemic hearts, and mitochondrial $\mathrm{H}_{2} \mathrm{O}_{2}$ production by reverse electron transfer (RET) is significantly decreased in TLR2 ${ }^{-/}$isolated heart mitochondria. a Mass spectroscopy identifies a peptide containing Cys260, which appears more frequently oxidized in WT myocardium compared with TLR2 ${ }^{-/-}$, in both postischemic (AR, area at risk) and remote (ANAR, area not at risk) myocardium $(n=3$ /group, $* P<0.05)$. b Isolated mitochondria from remote or post-ischemic myocardium isolated from TLR $2^{-/-}$animals after $30 \mathrm{~min}$ ischemia and 2 min reperfusion showed a reduced $\mathrm{H}_{2} \mathrm{O}_{2}$ production/ emission in presence of $5 \mathrm{mM}$ succinate $(n=3$, experiments were performed in duplicate and averaged, $* P<0.05$ ). Under these conditions ROS production occurs mainly at complex I through RET. The detected $\mathrm{H}_{2} \mathrm{O}_{2}$ emission can be completely suppressed by the uncoupler FCCP $(50 \mathrm{nM})$ and the complex II inhibitor atpenin A5 (Atp, $250 \mathrm{nM})$. The latter also excludes other ROS sources. The complex V inhibitor oligomycin (Oligo, $2 \mu \mathrm{g} / \mathrm{mL}$ ) increases the membrane potential and hence the ROS by RET. A difference in the ability to produce at the $\mathrm{Q}_{\mathrm{o}}$ site of complex III-induced by antimycin A (Ant, $1 \mu \mathrm{M}$ ) - was not observed, while the differential $\mathrm{H}_{2} \mathrm{O}_{2}$ emission in presence of the $\mathrm{Q}_{\mathrm{o}}$ site inhibitor stigmatellin (Stig, $1 \mu \mathrm{M}$ ) implies that TLR2 affects the ability for ROS production at the ubiquinone binding site of complex II. For details, see text

$\mathrm{III}_{\mathrm{Qo}}$ inhibitor stigmatellin reduced $\mathrm{H}_{2} \mathrm{O}_{2}$ production in WT and almost abrogated $\mathrm{H}_{2} \mathrm{O}_{2}$ production in TLR2 ${ }^{-/-}$mitochondria. While stigmatellin blocks the superoxide production at site $\mathrm{III}_{\mathrm{Qo}}$, site $\mathrm{II}_{\mathrm{Q}}$ can produce superoxide $/ \mathrm{H}_{2} \mathrm{O}_{2}$ under these conditions [22]. Our data indicate that also ROS production from this site is reduced in mitochondria of $\mathrm{TLR} 2^{-/-}$mice compared with WT mitochondria.

\section{Cx43 Phosphorylation Is Significantly Reduced in WT Hearts}

Phosphorylation of $\mathrm{Cx} 43$ regulates gap junction assembly and function. $\mathrm{Cx} 43$ phosphorylation can be visualized through its effect on the molecules' migration in electrophoretic gels. Figure 3 depicts a Western blot showing Cx43 in its dephosphorylated form in WT hearts compared with TLR2 ${ }^{-/}$hearts; the latter showing multiple bands attributed to different phosphorylation patterns.

\section{Patch-Clamp Recordings of Cx43-Transfected HL-1 Atrial Cells}

We tested the functional impact on TLR2-mediated signaling towards Cx43 and particularly the role of Cys 260 in a simplified model system. We studied the impact of TLR2 activation by $10 \mu \mathrm{g} / \mathrm{mL}$ bath application of TLR 2 agonist $\mathrm{Pam}_{3} \mathrm{CSK}_{4}$ on the spontaneous network activity in HL-1 atrial cell cultures transfected with either wild-type of mutant (C260A) Cx43 constructs with single-cell patch-clamp recordings of individual HL-1 cells. We selected those cultures, which under control conditions generated persistent and stable spontaneous pacemaker activity. Neither mean pacemaker frequency (mean frequency $(\mathrm{Hz}$, mean $\pm \mathrm{SEM})$ : $\mathrm{wtCx}=1.61 \pm 0.29$, $n=6$; mutCx $=1.34 \pm 0.34, n=6 ; P=0.56)$ nor variability (coefficient of variation $(\mathrm{CV})$ : $\mathrm{wtCx}=0.15 \pm 0.04, n=6$; mutCx $=0.21 \pm 0.09, n=6 ; P=0.57$ ) nor maximal depolarization speed of action potentials $((\max d V / d t, \mathrm{mV} / \mathrm{ms})$ : ${ }_{\mathrm{wtCx}}=3.9 \pm 0.9, n=6 ;$ mutCx $\left.=6.3+2.1, n=6 ; P=0.33\right)$ was different between HL-1 cultures transfected with wildtype or mutant $\mathrm{Cx} 43$ constructs under control conditions in the absence of TLR2 activation. After at least $5 \mathrm{~min}$ stable baseline activity, we applied $10 \mu \mathrm{g} / \mathrm{mL} \mathrm{Pam}_{3} \mathrm{CSK}_{4}$ to activate TLR2 and recorded the next $5 \mathrm{~min}$ in the presence of the agonist. While action potential properties were not significantly altered by TLR2 for both wild-type and mutant $\mathrm{Cx} 43$ transfected HL-1 cultures, we detected a significant difference between the TLR2-mediated changes in the mean pacemaker frequency: in HL-1 cultures transfected with wild-type $\mathrm{Cx} 43$, we detected a small reduction $(\sim 10 \%)$ of mean discharge frequencies. In contrast, the mean frequency during TLR2 activation in HL-1 cultures transfected with mutant (C260A) $\mathrm{Cx} 43$ was about $20 \%$ faster compared with cultures expressing wild-type connexin (mean frequency in TLR2 compared with control $(100 \%)$ : $w t C x 43=91.4 \pm 7.4, n=4$; $\operatorname{mutCx} 43=$ $112.4 \pm 6.1, n=4 ; P=0.03$; Fig. 4). Consistent with the different functional properties of wild-type and mutant $\mathrm{Cx} 43$, we observed different connectivity between HL-1 cells when probed by neurobiotin dialysis via the patch pipette in the 
Fig. 3 Cx43 phosphorylation is significantly decreased in WT myocardium after $\mathrm{MI} / \mathrm{R}$. Phosphorylation of $\mathrm{Cx} 43$ changes migration in electrophoretic gels $(n=6 /$ group $)$

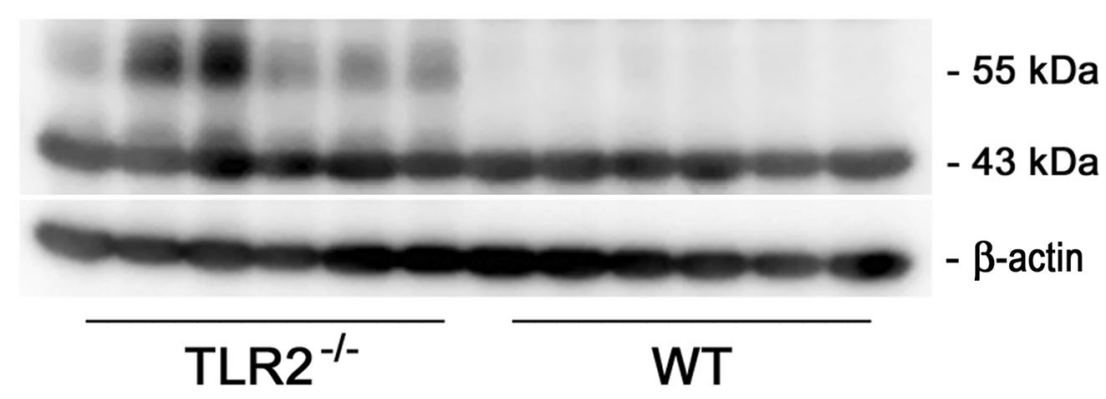

recorded HL-1 cardiomyocytes and post hoc processing with fluorescently labeled streptavidin. While neurobiotin moved to a large number of direct and indirect neighboring cells in cultures expressing wild-type Cx43 (Fig. 4a), it remained local and stained only immediate neighbors of the patched cell in HL-1 cultures, which expressed the mutant (C260A) Cx43 (Fig. 4b).

\section{Discussion}

Murine $\mathrm{MI} / \mathrm{R}$ electrical conduction in the myocardium is faster in TLR2-/- mice, compared with WT. This is associated with reduced mitochondrial ROS formation which subsequently leads to reduced oxidation of $\mathrm{Cx} 43$ at site Cys260. Cx43 C260A mutation in HL-1 cardiomyocytes modifies the TLR2-dependent alteration of cardiomyocyte coupling and action potential propagation.

Regulation of gap junction communication remainsowing to its complexity-incompletely understood. Opening, coupling, gap junction formation, and subcellular location of $\mathrm{Cx} 43$ have been extensively studied putting forward phosphorylation at least 19 phosphorylation sites as the main regulatory mechanism for gap junction communication. Less attention has been paid to various cysteine residues contained within its amino acid sequence that might be subject

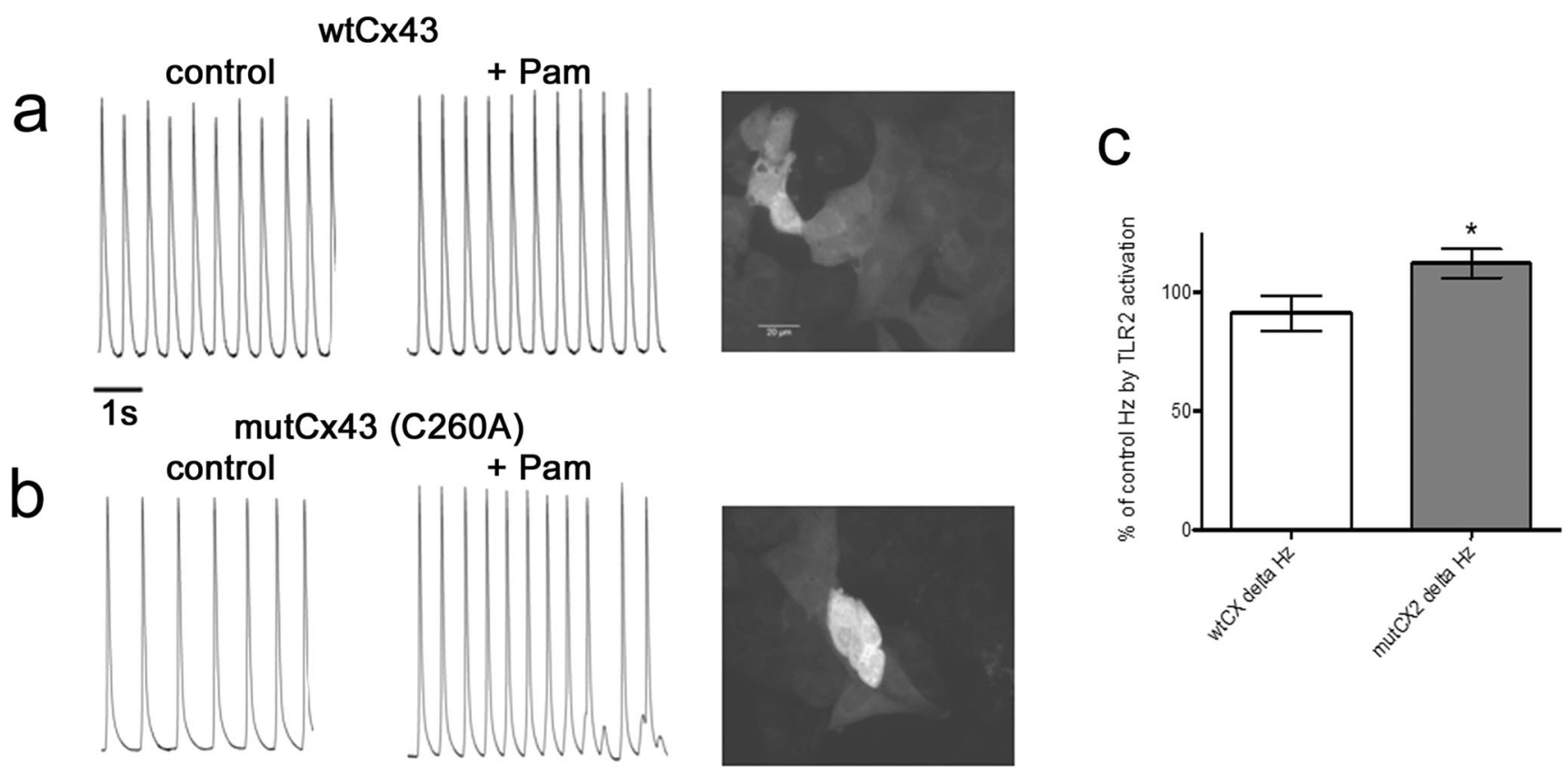

\section{$\overline{1 s}$}

Fig. 4 Expression of mutant C260A Cx43 in atrial HL-1 cultures accelerates pacemaker activity in response to TLR2 activation. a Currentclamp recordings of a single HL-1 atrial cell in a pacemaking culture transfected with wild-type Cx43 under control conditions (left panel) and in the presence of TLR2 ligand $\mathrm{Pam}_{3} \mathrm{CSK}_{4}(10 \mu \mathrm{g} / \mathrm{mL})$. Right panel shows post hoc confocal analysis of gap junction coupling of the recorded HL-1 cell via neurobiotin dialysis during patch-clamp recording and post hoc processing with fluorescently labeled streptavidin. b Current-clamp recordings of a single HL-1 atrial cell in a pacemaking culture transfected with mutant (C260A) Cx43 under control conditions (left panel) and in the presence of $10 \mu \mathrm{g} / \mathrm{mL} \mathrm{Pam}{ }_{3} \mathrm{CSK}_{4}$ for TLR2 activation (middle panel). Right panel shows post hoc confocal analysis of gap junction coupling of the recorded HL-1 cell via neurobiotin dialysis. Note acceleration of pacemaker frequency by TLR2 receptor activation. c Mean relative pacemaker frequency change by TLR 2 receptor activation is significantly higher in mutant (C260A) Cx43 expressing HL-1 cardiomyocytes $(n=4$ / group, $* P<0.05$ ) 
to redox regulation especially in the situation of ischemia/reperfusion. Toyofuku et al. studied cysteine residues within the extracellular loops of $\mathrm{Cx} 43$ and observed the mutations of Cys54, Cys187, Cys192, and Cys198 to Ser. These mutations resulted in electrical uncoupling and failure of $\mathrm{Ca}^{2+}$ propagation to neighboring cells [23]. Furthermore, $\mathrm{Cx} 43$ hemichannel opening was increased by intracellular application of the reducing agent DTT. This suggests redox-sensitive sites within the cytoplasmic tail or loop that decrease opening probability when oxidized [24]. But despite numerous studies defining the general effect of redox potential on gap junction and hemichannel opening, little is known about the redox sensors within the molecule [8].

The oxidation site identified in our study is Cys260, located in the $\mathrm{C}$-terminal cytoplasmic tail of $\mathrm{Cx} 43$. Truncation of the C-terminal tail, a process that also occurs under stress conditions [25], affects fast gating [26] and abolishes $\mathrm{pH}-$ dependent uncoupling of $\mathrm{Cx} 43$ gap junctions [27]. The proposed mechanism of action is an interaction between the $\mathrm{C}$ tail and a yet unidentified target within the pore, possibly the cytoplasmic loop of $\mathrm{Cx} 43$. Oxidation at Cys 260 might affect this interaction. Besides, Cys260 is situated in close proximity to three phosphorylation sites: Ser255, Ser262, and Tyr265. All have been related to gap junction coupling and function (reviewed by Solan et al. [28]). Kinases identified, that phosphorylate $\mathrm{Cx} 43$ at those sites, are Src (at Tyr265), leading to disruption of gap junction communication [29], and MAPK (at Ser255 and Ser262) [30]. Solan and Lampe described that phosphorylation of $\mathrm{Cx} 43$ affects its migration in gels [28]. We have observed a single band of (unphosphorylated) $\mathrm{Cx} 43$ in post-ischemic WT myocardium compared with multiple bands in TLR $2^{-/}$myocardium; the latter can be attributed to different phosphorylation states of the molecule. Description of the very sites of phosphorylation will warrant further studies [28].

We have identified mitochondrial ROS production within the respiratory chain as the origin of oxidizing agents most probably responsible for the increased oxidation rate of $\mathrm{Cx} 43$ during MI/R. It is well established that the mitochondrial succinate concentration increases during ischemia. This drives reverse electron transfer to complex I and the accompanying deleterious oxidative burst during reperfusion [15-18]. The observed decrease in the $\mathrm{H}_{2} \mathrm{O}_{2}$ emission of heart mitochondria from TLR2 ${ }^{-/-}$mice could be caused either by a decreased production at complex I-which can be realized, e.g., by a stabilization of the D-form of complex I [17]—or by an increased activity of the antioxidant systems in the matrix. In addition, we observed that also the ability to produce ROS at site $\mathrm{II}_{\mathrm{Q}}$ was decreased while that at site $\mathrm{III}_{\mathrm{Qo}}$ was unaffected by the TLR2 knockout. However, the exact molecular mechanism on how TLR2 affects the mitochondrial ROS production or ROS scavenging activity needs to be established in future investigations.
To further investigate the consequences of TLR2dependent Cx43 oxidation at Cys260, we used HL-1 cardiomyocytes transfected with WT or C260A Cx43. Although primary (ventricular) cardiomyocytes would have more closely resembled the arrhythmia phenotype observed in vivo, we decided to use the HL-1 cell model to allow for lentiviral transfection.

In this simplified model, we were able to demonstrate that TLR2 signaling targets Cys 260 in $\mathrm{Cx} 43$ and that its modification has functional consequences for the network pacemaker activity of atrial cells. In particular, its regulation appears to help stabilize network activity during TLR2 signaling and thus prevents frequency enhancement as observed in atrial networks expressing the mutant $\mathrm{C} 260 \mathrm{~A} \mathrm{Cx} 43$. However, investigations in primary cardiomyocytes, whole-heart or in vivo models, will be required to further understand the mechanisms and clinical relevance of innate immune receptor TLR2 signaling on cardiac arrhythmia development.

\section{Conclusion}

In conclusion, reversible oxidation of $\mathrm{Cx} 43$ at $\mathrm{Cys} 260$ represents a novel mechanism by which $\mathrm{Cx} 43$ phosphorylation and gap junction communication between cardiomyocytes can be regulated. This regulation is dependent upon TLR2 signaling.

Acknowledgments HL-1 mouse atrial cardiomyocytes were a kind gift from William C Claycomb.

Source of Funding This work was supported by a grant of the Deutsche Forschungsgemeinschaft (SFB815 A17 to J.M. and K.Z.; SFB815 A02 to S.D.; SFB815 A01 to R.B.; SFB815 A13 to J.R.; and SFB815 Z01 to I.W.). SFB stands for SonderForschungsBereich.

\section{Compliance with Ethical Standards}

Conflicts of Interest The authors declare that they have no conflict of interest.

Ethical Approval All applicable international, national, and/or institutional guidelines for the care and use of animals were followed.

\section{References}

1. Leemans, J. C., Stokman, G., Claessen, N., Rouschop, K. M., Teske, G. J. D., Kirschning, C. J., Akira, S., van der Poll, T., Weening, J. J., \& Florquin, S. (2005). Renal-associated TLR2 mediates ischemia/reperfusion injury in the kidney. The Journal of Clinical Investigation, 115(10), 2894-2903.

2. Mersmann, J., Iskandar, F., Latsch, K., Habeck, K., Sprunck, V., Zimmermann, R., Schumann, R. R., Zacharowski, K., \& Koch, A. (2013). Attenuation of myocardial injury by HMGB1 blockade during ischemia/reperfusion is toll-like receptor 2-dependent. Mediators of Inflammation, 2013, 174168. 
3. Sakata, Y., Dong, J.-W., Vallejo, J. G., Huang, C.-H., Baker, J. S., Tracey, K. J., Tacheuchi, O., Akira, S., \& Mann, D. L. (2007). Tolllike receptor 2 modulates left ventricular function following ischemia-reperfusion injury. American Journal of Physiology. Heart and Circulatory Physiology, 292(1), H503-H509.

4. Zhang, J.-X., Wu, H.-S., Wang, H., Zhang, J.-H., Wang, Y., \& Zheng, Q.-C. (2005). Protection against hepatic ischemia/ reperfusion injury via downregulation of toll-like receptor 2 expression by inhibition of Kupffer cell function. World Journal of Gastroenterology, 11(28), 4423-4426.

5. Mersmann, J., Koch, A., Tran, N., Zimmermann, R., Granja, T. F., Larmann, J., Herzog, C., Theilmeier, G., Bornstein, S. R., Kirschning, C. J., \& Zacharowski, K. (2010). Toll-like receptor 2 signaling triggers fatal arrhythmias upon myocardial ischemia-reperfusion. Critical Care Medicine, 38(10), 1927-1932.

6. Erridge, C. (2010). Endogenous ligands of TLR2 and TLR4: agonists or assistants? Journal of Leukocyte Biology, 87(6), 989-999.

7. Mersmann, J., Tran, N., Latsch, K., Habeck, K., Iskandar, F., Zimmermann, R., \& Zacharowski, K. (2012). Akt or phosphoinositide-3-kinase inhibition reverses cardio-protection in toll-like receptor 2 deficient mice. Resuscitation, 83(11), 14041410.

8. Retamal, M. A. (2014). Connexin and pannexin hemichannels are regulated by redox potential. Frontiers in Physiology, 5, 80.

9. Petric, S., Clasen, L., van Wessel, C., Geduldig, N., Ding, Z., Schullenberg, M., Mersmann, J., Zacharowski, K., Aller, M. I., Schmidt, K. G., \& Donner, B. C. (2012). In vivo electrophysiological characterization of TASK-1 deficient mice. Cellular Physiology and Biochemistry, 30(3), 523-537.

10. Dröse, S., Brandt, U., \& Hanley, P. J. (2006). K+-independent actions of diazoxide question the role of inner membrane KATP channels in mitochondrial cytoprotective signaling. The Journal of Biological Chemistry, 281(33), 23733-23739.

11. Bleier, L., Wittig, I., Heide, H., Steger, M., Brandt, U., \& Dröse, S. (2015). Generator-specific targets of mitochondrial reactive oxygen species. Free Radical Biology \& Medicine, 78, 1-10.

12. Leichert, L. I., Gehrke, F., Gudiseva, H. V., Blackwell, T., Ilbert, M., Walker, A. K., Strahler, J. R., Andrews, P. C., \& Jakob, U. (2008). Quantifying changes in the thiol redox proteome upon oxidative stress in vivo. Proceedings of the National Academy of Sciences of the United States of America, 105(24), 8197-8202.

13. Wilson, A. A., Kwok, L. W., Hovav, A.-H., Ohle, S. J., Little, F. F., Fine, A., \& Kotton, D. N. (2008). Sustained expression of alpha1antitrypsin after transplantation of manipulated hematopoietic stem cells. American Journal of Respiratory Cell and Molecular Biology, 39(2), 133-141.

14. Mersmann, J., Berkels, R., Zacharowski, P., Tran, N., Koch, A., Iekushi, K., Dimmeler, S., Granja, T. F., Boehm, O., Claycomb, W. C., \& Zacharowski, K. (2010). Preconditioning by toll-like receptor 2 agonist Pam3CSK4 reduces CXCL1-dependent leukocyte recruitment in murine myocardial ischemia/reperfusion injury. Critical Care Medicine, 38(3), 903-909.

15. Chouchani, E. T., Pell, V. R., Gaude, E., Aksentijević, D., Sundier, S. Y., Robb, E. L., Logan, A., Nadtochiy, S. M., Ord, E. N. J., Smith, A. C., Eyassu, F., Shirley, R., et al. (2014). Ischaemic accumulation of succinate controls reperfusion injury through mitochondrial ROS. Nature, 515(7527), 431-435.

16. Brand, M. D. (2016). Mitochondrial generation of superoxide and hydrogen peroxide as the source of mitochondrial redox signaling. Free Radical Biology \& Medicine, 100, 14-31.
17. Dröse, S., Stepanova, A., \& Galkin, A. (2016). Ischemic A/D transition of mitochondrial complex I and its role in ROS generation. Biochimica et Biophysica Acta, 1857(7), 946-957.

18. Votyakova, T. V., \& Reynolds, I. J. (2008). $\Delta \Psi$ m-dependent and independent production of reactive oxygen species by rat brain mitochondria. Journal of Neurochemistry, 79(2), 266-277.

19. Quinlan, C. L., Orr, A. L., Perevoshchikova, I. V., Treberg, J. R., Ackrell, B. A., \& Brand, M. D. (2012). Mitochondrial complex II can generate reactive oxygen species at high rates in both the forward and reverse reactions. The Journal of Biological Chemistry, 287(32), 27255-27264.

20. Siebels, I., \& Dröse, S. (2013). Q-site inhibitor induced ROS production of mitochondrial complex II is attenuated by TCA cycle dicarboxylates. Biochim Biophys Acta - Bioenerg, 1827(10), 11561164.

21. Bleier, L., \& Dröse, S. (2013). Superoxide generation by complex III: from mechanistic rationales to functional consequences. Biochim Biophys Acta - Bioenerg, 1827(11), 1320-1331.

22. Bonke, E., Zwicker, K., \& Dröse, S. (2015). Manganese ions induce $\mathrm{H} 2 \mathrm{O} 2$ generation at the ubiquinone binding site of mitochondrial complex II. Archives of Biochemistry and Biophysics, 580, 75-83.

23. Toyofuku, T., Yabuki, M., Otsu, K., Kuzuya, T., Hori, M., \& Tada, M. (1998). Intercellular calcium signaling via gap junction in connexin-43-transfected cells. The Journal of Biological Chemistry, 273(3), 1519-1528.

24. Retamal, M. A., Schalper, K. A., Shoji, K. F., Bennett, M. V. L., \& Sáez, J. C. (2007). Opening of connexin 43 hemichannels is increased by lowering intracellular redox potential. Proceedings of the National Academy of Sciences of the United States of America, 104(20), 8322-8327.

25. Joshi-Mukherjee, R., Coombs, W., Burrer, C., de Mora, I. A., Delmar, M., \& Taffet, S. M. (2009). Evidence for the presence of a free C-terminal fragment of cx43 in cultured cells. Cell Communication \& Adhesion, 14(2-3), 75-84.

26. Moreno, A. P., Chanson, M., Anumonwo, J., Scerri, I., Gu, H., Taffet, S. M., \& Delmar, M. (2002). Role of the carboxyl terminal of connexin43 in transjunctional fast voltage gating. Circulation Research, 90(4), 450-457.

27. Morley, G. E., Ek-Vitorin, J. F., Taffet, S. M., \& Delmar, M. (1997). Structure of connexin 43 and its regulation by $\mathrm{pH}$ i. Journal of Cardiovascular Electrophysiology, 8(8), 939-951.

28. Solan, J. L., \& Lampe, P. D. (2014). Specific Cx43 phosphorylation events regulate gap junction turnover in vivo. FEBS Letters, 588(8), $1423-1429$.

29. Lin, R., Warn-Cramer, B. J., Kurata, W. E., \& Lau, A. F. (2001). vSrc phosphorylation of connexin 43 on Tyr247 and Tyr265 disrupts gap junctional communication. The Journal of Cell Biology, 154(4), 815-827.

30. Doble, B. W., Dang, X., Ping, P., Fandrich, R. R., Nickel, B. E., Jin, Y., Cattini, P. A., \& Kardami, E. (2004). Phosphorylation of serine 262 in the gap junction protein connexin-43 regulates DNA synthesis in cell-cell contact forming cardiomyocytes. Journal of Cell Science, 117(3), 507-514.

Publisher's Note Springer Nature remains neutral with regard to jurisdictional claims in published maps and institutional affiliations. 